\title{
General interpolation scheme for thermal fluctuations in superconductors
}

\author{
L. Marotta, M. Camarda, G. G. N. Angilella, and F. Siringo \\ Dipartimento di Fisica e Astronomia, Università di Catania, INFN Sezione di Catania, Laboratorio MATIS-INFM and CNISM Sez. di \\ Catania, Via S. Sofia 64, I-95123 Catania, Italy
}

(Received 25 July 2005; revised manuscript received 13 February 2006; published 29 March 2006)

\begin{abstract}
We present a general interpolation theory for the phenomenological effects of thermal fluctuations in superconductors. Fluctuations are described by a simple gauge invariant extension of the Gaussian effective potential for the Ginzburg-Landau static model. The approach is shown to be a genuine variational method, and to be stationary for infinitesimal gauge variations around the Landau gauge. Correlation and penetration lengths are shown to depart from the mean field behavior in a more or less wide range of temperature below the critical regime, depending on the class of material considered. The method is quite general and yields a very good interpolation of the experimental data for very different materials.
\end{abstract}

DOI: 10.1103/PhysRevB.73.104517

\section{INTRODUCTION}

In a recent paper $^{1}$ we have shown that the Gaussian effective potential (GEP) can describe the behavior of superconductors, thus allowing for a comparison with the experimental data. The GEP has a long history ${ }^{2-11}$ and has been discussed by several authors as a tool for describing the breaking of symmetry in the standard model of electroweak interactions, ${ }^{11,12}$ but in that context no real comparison with experimental data will be achievable until the detection of the Higgs boson. Thus High $T_{c}$ superconductors represent the best way to test the reliability of the method. While the comparison with experimental data is of special importance as a test for the GEP variational method itself, the method can be regarded as a general interpolation scheme for the precritical region of superconductors: too close to the critical point for thermal fluctuations being negligible, but not yet in the critical range where universality sets in. In this precritical range, the GEP provides an interpolation between the mean-field behavior and the critical limit. Besides standard phenomenological parameters the method relies on one free parameter which characterizes the width of the precritical range and allows for a very good fit of the experimental data for very different materials ranging from cuprates like $\mathrm{YBa}_{2} \mathrm{Cu}_{2} \mathrm{O}_{7-\delta}$ to the double band superconductor $\mathrm{MgB}_{2}$. The free parameter turns out to be a characteristic energy scale of the material.

The general phenomenology of superconductivity can be described by the standard Anderson-Higgs mechanism: The supercurrent is carried by pairs of charged fermions whose nonvanishing expectation value breaks the gauge symmetry, and endows the gauge bosons with a nonzero mass. Thus, the standard Ginzburg-Landau (GL) effective Lagrangian provides the best framework for a general description of the phenomenology. Moreover, as the GL action can be seen as a power expansion of the exact action around the critical point, the GL action must be recovered by any microscopic theory at least around the transition. Therefore, whatever the origin of the microscopic pairing mechanism, the GL action is a sound starting point for a general interpolation scheme of superconductivity. Of course we cannot trust the mean-field approach to the GL effective theory, and we expect that in high $T_{c}$ superconductors many unconventional properties should have to do with the breaking down of the simple
PACS number(s): 74.20.De, 74.25.Bt, 11.15.Tk

mean-field picture. Actually, unconventional superconductors are characterized by a very small correlation length $\xi$, which allows the experimentalists to get closer to the critical point where the thermal fluctuations cannot be neglected and the mean-field approximation is doomed to fail. As far as we know, there is no clear evidence that the critical universal behavior can always be reached in the cuprate superconductors: A weakly charged superfluid regime has been predicted and observed ${ }^{13-16}$ in nearly optimally doped samples, while a charged critical behavior has been reported for the underdoped samples. ${ }^{17}$ Recently the superconducting pyrochlores have been shown to open up a window onto the charged critical regime. ${ }^{18}$ However, it is out of doubt that an intermediate range of temperature is now accessible, where thermal fluctuations are not negligible even when the sample is still out of the truly critical regime. Thus, in order to describe some unconventional properties of high $T_{c}$ superconductors, we need to incorporate the role of thermal fluctuations, but unfortunately we cannot rely on the standard renormalization group methods ${ }^{19}$ which would only describe the limiting universal behavior. We need an approximate approach to the GL action for the nonuniversal regime where the behavior depends on the physical parameters of the sample, and we would prefer a nonperturbative approximation in order to deal with any coupling, whatever its strengthness.

As a toy model for electroweak interactions, the scalar electrodynamics in four dimensions has been studied by Ibañez-Meier et $a l .{ }^{12}$ who computed the GEP by use of Cartesian coordinates for the field components. This choice breaks the $U(1)$ symmetry of the field and gives rise to an unphysical, and undesirable, massive degree of freedom. However, in three space dimensions, the $U(1)$ scalar electrodynamics is equivalent to the standard static GL effective model of superconductivity, and the GEP becomes a variational tool for superconductivity. In this paper, we show that the unphysical degree of freedom can be avoided by use of polar coordinates for the field components. Moreover, the choice of a $U(1)$ symmetric Gaussian functional restores the gauge symmetry and makes the theory gauge invariant for infinitesimal gauge changes around the Landau gauge. In a previous work we discussed the same approximation in unitarity gauge. ${ }^{1}$ The method has been extended to twodimensional systems by Abreu et al. ${ }^{20}$ in order to study film 
superconductors. Unfortunately, we found out that the claimed exact ${ }^{21}$ integration of the longitudinal component of the electromagnetic vector field $A_{\mu}$ is only approximatively exact. Actually, as we show in the present paper, that approximation is a variational one and its accuracy can be checked by standard variational arguments. Indeed, by a direct comparison of the energies, we show that the present method yields a better result compared with the Cartesian coordinate GEP of Ibañez-Meier et al. ${ }^{12}$

The variational method provides a way to evaluate important phenomenological quantities like the correlation length $\xi$ and the penetration depth $\lambda$ that emerge as the solution of a set of coupled equations. The GL parameter $\kappa_{\mathrm{GL}}=\lambda / \xi$, whose critical behavior has been addressed by Herbut and Tesanovic, ${ }^{19}$ is here shown to be described by a very simple relation in the precritical regime where it is found to be temperature dependent, in contrast to the mean-field result. Thus, the GL parameter has been chosen as a useful measure of thermal fluctuations: Its dependence on temperature is a sign of the breaking down of the mean-field approximation which predicts a constant $\kappa_{G L}$. Unfortunately, experimental data on the temperature dependence of the GL parameter are quite fragmentary in the literature. The behavior of $\xi$ and $\lambda$ can be extracted by the knowledge of the critical magnetic fields, but experimental limits on this data have narrowed our chances of a wide comparison with experiments. Up to now, our GEP has shown the capability of fitting the GL parameter for all the materials that we have been able to test.

The paper is organized as follows: In Sec. II the GL action and partition function are reported and the symmetry of the model is discussed together with the conditions for the gauge invariance of the variational functional; In Sec. III the integration of the longitudinal vector field is shown to be a variational approximation; In Sec. IV the GEP is evaluated in polar coordinates and the result is compared with the Cartesian coordinate method of Ref. 12; In Sec. V the variational equations are solved for some set of phenomenological parameters and the GL parameter is compared with the available experimental data; some comments and final remarks on the interpolation method are reported.

\section{THE GL ACTION AND GAUGE INVARIANCE}

Let us consider the standard static GL action ${ }^{22}$

$$
\begin{aligned}
S= & \int d^{3} x\left[\frac{1}{4} F_{\mu \nu} F^{\mu \nu}+\frac{1}{2}\left(D_{\mu} \phi\right)^{*}\left(D^{\mu} \phi\right)\right. \\
& \left.+\frac{1}{2} m_{B}^{2} \phi^{*} \phi+\lambda_{B}\left(\phi^{*} \phi\right)^{2}\right] .
\end{aligned}
$$

Here $\phi$ is a complex (charged) scalar field, its covariant derivative is defined according to

$$
D_{\mu}=\partial_{\mu}+i e_{B} A_{\mu}
$$

and $\mu, \nu=1,2,3$ run over the three space dimensions. The components of the magnetic field $F_{\mu \nu}=\partial_{\mu} A_{\nu}-\partial_{\nu} A_{\mu}$ satisfy

$$
\frac{1}{2} F_{\mu \nu} F^{\mu \nu}=|\nabla \times \mathbf{A}|^{2}
$$

and the partition function is defined by the functional integral

$$
Z=\int D\left[\phi, \phi^{*}, A_{\mu}\right] e^{-S} .
$$

The action $S$ in Eq. (1) has a local $U(1)$ symmetry as it is invariant for a local gauge transformation

$$
\begin{aligned}
& \mathbf{A} \rightarrow \mathbf{A}+\nabla \chi(x) \\
& \phi \rightarrow \phi e^{-i e_{B} \chi(x)},
\end{aligned}
$$

where $\chi(x)$ is an arbitrary function. The integration over $\mathbf{A}$ is then redundant in Eq. (4) and a gauge fixing term must be inserted in order to make the partition function finite. According to the standard De Witt-Faddeev-Popov method ${ }^{23}$ the partition function can be written as

$$
Z=\int D\left[\phi, \phi^{*}, A_{\mu}\right] e^{-S} e^{-S_{f i x}},
$$

where the gauge fixing action is

$$
S_{f i x}=\int d^{3} x \frac{1}{2 \epsilon} f^{2}
$$

and $f(A)=0$ is an arbitrary gauge constraint. $Z$ is invariant for any change of the parameter $\epsilon$ and of the constraint $f$. With some abuse of language, this invariance property is referred to as gauge invariance while it is a more general invariance as $Z$ does not depend on the shape of the weight factor which has been added in Eq. (7). Only for $\epsilon \rightarrow 0$ the weight factor $\exp \left(-S_{f i x}\right)$ becomes a $\delta$ function which enforces the constraint $f=0$ on the vector field $\mathbf{A}$. Thus gauge invariance denotes the invariance of the theory for any change of the constraint $f=0$ in the limit $\epsilon \rightarrow 0$. This is a weaker condition, but unfortunately even this is not fulfilled by some approximations to the partition function. In some approximation schemes, such as the perturbative method, the action $S$ is split in two parts $S=S_{0}+S_{1}$ and different approximations are considered for the two parts: For instance $S_{0}$ might need no approximations while $\exp \left(-S_{1}\right)$ could be expanded as a power series of some small parameter. Even when $S$ is gauge invariant the approximate $Z$ can result to be gauge dependent. It is easy to show that if both $S_{0}$ and $S_{1}$ are gauge invariant then the approximate $Z$ is gauge invariant in the sense that in the limit $\epsilon \rightarrow 0$ any gauge change $f \rightarrow f+\delta f$ leaves the approximate $Z$ unchanged. Thus, in order to obtain a gauge invariant treatment, we must take care that the exact action $S$ is split in gauge invariant terms.

The GEP is a sort of optimized first-order approximation: In Cartesian coordinates ${ }^{12}$ the action is split according to

$$
\begin{aligned}
S_{0}= & \int d^{3} x\left[\frac{1}{4} F_{\mu \nu} F^{\mu \nu}+\frac{1}{2}\left(\partial_{\mu} \phi_{1}\right)\left(\partial^{\mu} \phi_{1}\right)+\frac{1}{2}\left(\partial_{\mu} \phi_{2}\right)\left(\partial^{\mu} \phi_{2}\right)\right. \\
& \left.+\frac{1}{2} \Omega_{1}^{2}\left(\phi_{1}-\varphi_{1}\right)^{2}+\frac{1}{2} \Omega_{2}^{2}\left(\phi_{2}-\varphi_{2}\right)^{2}+\frac{1}{2} \Delta^{2} \mathbf{A}^{2}\right],
\end{aligned}
$$

where $\phi_{1}+i \phi_{2}=\phi$ and $S_{1}=S-S_{0}$. Here $\Omega_{i}, \Delta$, and $\varphi_{i}$ are arbitrary variational parameters, and the free energy is evaluated up to first order in the expansion of $S_{1}$. The result is then optimized by variation of the free parameters. When the shifts $\varphi_{i}$ are nonvanishing the action $S_{0}$ is not $U(1)$ invariant, 
and the approximate partition function fails to be gauge invariant. The lack of symmetry is evident from the appearance of an unphysical massive Goldstone boson (in fact, there are two different mass parameters $\Omega_{1}$ and $\Omega_{2}$ ). In the next section we show by a variational argument that in polar coordinates the action $S_{0}$ can be taken as

$$
\begin{aligned}
S_{0}= & \int d^{3} x\left[\frac{1}{4} F_{\mu \nu} F^{\mu \nu}+\frac{1}{2}\left(\partial_{\mu} \rho\right)\left(\partial^{\mu} \rho\right)\right. \\
& \left.+\frac{1}{2} \Omega^{2}(\rho-\varphi)^{2}+\frac{1}{2} \Delta^{2} \mathbf{A}^{2}\right],
\end{aligned}
$$

where the field is decomposed according to $\phi=\rho \exp (i \gamma)$ and $\rho$ is a $U(1)$ invariant real field. The explicit integration of the phase $\gamma$ makes the action invariant for rotations in the $\phi_{1}, \phi_{2}$ plane. As before $\Omega, \Delta$ and the shift $\varphi$ are variational parameters. We still have a noninvariant term quadratic in the vector field. However, if we take

$$
f=\partial_{\mu} A^{\mu}
$$

any infinitesimal gauge change $f \rightarrow f+\delta f$ yields

$$
A_{\mu} \rightarrow A_{\mu}+\partial_{\mu} \chi
$$

and up to a surface term

$$
A^{\mu} A_{\mu} \rightarrow A^{\mu} A_{\mu}-2 \chi f+\mathcal{O}\left(\delta f^{2}\right)
$$

so that for $\epsilon \rightarrow 0$ the constraint $f=0$ restores gauge invariance up to first order in the gauge change around the Landau gauge $\partial_{\mu} A^{\mu}=0$. This discussion motivates in part the choice of polar coordinates that gives rise to an explicitly rotational invariant variational functional and no unphysical massive Goldstone boson. Another motivation arises from the more pragmatic observation that the free energy is lower with the choice of this polar coordinate variational functional, and since we show that both methods are genuine variational methods a lower free energy means a higher accuracy of the results.

\section{GL ACTION IN POLAR COORDINATES}

In the previous section we have given some motivations for the choice of polar coordinates in Landau gauge. In three space dimensions that gauge is the transverse gauge $f=\boldsymbol{\nabla} \cdot \mathbf{A}$. A simple coordinate change $\phi \rightarrow \rho \exp (i \gamma)$ in the partition function Eq. (7) yields

$$
Z=\int D\left[A_{\mu}, \rho^{2}\right] e^{-\int d^{3} x \mathcal{L}} \int D[\gamma] e^{-\int d^{3} x \mathcal{L}_{\gamma}},
$$

where $\mathcal{L}$ is the phase independent Lagrangian

$$
\begin{aligned}
\mathcal{L}= & \frac{1}{2}(\boldsymbol{\nabla} \times \mathbf{A})^{2}+\frac{1}{2} \partial_{\mu} \rho \partial^{\mu} \rho+\frac{1}{2} m_{B}^{2} \rho^{2}+\lambda_{B} \rho^{4} \\
& +\frac{1}{2} e_{B}^{2} \rho^{2} \mathbf{A}^{2}+\frac{1}{2 \epsilon}(\boldsymbol{\nabla} \cdot \mathbf{A})^{2}
\end{aligned}
$$

and $\mathcal{L}_{\gamma}$ is the sum of the Lagrangian terms which depend on the phase $\gamma$

$$
\mathcal{L}_{\gamma}=\frac{1}{2} \rho^{2} \partial_{\mu} \gamma \partial^{\mu} \gamma+e_{B} \rho^{2} \partial_{\mu} \gamma A^{\mu} .
$$

If the last term of $\mathcal{L}_{\gamma}$ were neglected, the phase $\gamma$ could be integrated exactly (the integral is Gaussian). It has been claimed $^{1,20,21}$ that in transverse gauge that term should vanish exactly: The argument was that in transverse gauge there is no longitudinal component of the vector field. In fact, the Fourier transform of the gradient $\partial_{\mu} \gamma$ is proportional to the wave vector $\mathbf{k}$, and this is orthogonal to the Fourier transform of $\mathbf{A}$ for the gauge constraint $f(\mathbf{k})=\mathbf{k} \cdot \mathbf{A}(\mathbf{k})=0$. However, this holds only for Fourier transforms. The last term of $\mathcal{L}_{\gamma}$ contains a $\rho^{2}$ factor which is not constant, and thus, in this case, the space integral of this term in the action cannot be replaced by the simple inverse-space integral of the product of the Fourier transforms (we could do that if the factor $\rho$ were constant). Thus, we cannot neglect that term and the exact integration over the phase $\gamma$ would depend on the field A. We stress that any gauge change does not solve the problem either, as an undesirable longitudinal component of the field $\mathbf{A}$ would take the place of the phase $\gamma$. For instance, in Ref.24 the phase $\gamma$ is gauged away but the quadratic term $e_{B}^{2} \rho^{2} \mathbf{A}^{2}$ contains an implicit coupling term $e_{B}^{2} \rho^{2} \mathbf{A}_{L} \cdot \mathbf{A}_{T}$ between the transversal component $\mathbf{A}_{T}$ and the longitudinal component $\mathbf{A}_{L}$ of the vector field. Again the integral of this coupling term would vanish if $\rho$ were constant (as it is obvious by the same Fourier transform argument discussed above), but it is not vanishing in general and prevents from an exact integration of the longitudinal component.

We can get rid of the phase by a variational argument: It turns out that the neglection of the phase terms is not an exact integration, but a good variational approximation. Let us denote by $\mathcal{L}_{0}$ the phase dependent Lagrangian without the last term

$$
\mathcal{L}_{0}=\mathcal{L}_{\gamma}\left(e_{B}=0\right)=\frac{1}{2} \rho^{2} \partial_{\mu} \gamma \partial^{\mu} \gamma
$$

We observe that up to constant factors the exact integration over $\gamma$ yields

$$
\int D[\gamma] e^{-\int d^{3} x \mathcal{L}_{0}} \sim \prod_{x} \frac{1}{\rho} .
$$

Thus, we may write the $D\left[\rho^{2}\right]$ integral in Eq. (14) as

$$
Z \sim \int D\left[A_{\mu}, \rho\right] e^{-\int d^{3} x \mathcal{L}}\left\{\frac{\int D[\gamma] e^{-\int d^{3} x \mathcal{L}_{\gamma}}}{\int D[\gamma] e^{-\int d^{3} x \mathcal{L}_{0}}}\right\} .
$$

We define the average over the phase as

$$
\langle(\ldots)\rangle_{\gamma}=\frac{\int D[\gamma] e^{-\int d^{3} x \mathcal{L}_{0}}(\ldots)}{\int D[\gamma] e^{-\int d^{3} x \mathcal{L}_{0}}} .
$$

With this notation the exact partition function Eq. (14) reads 


$$
Z=\int D\left[A_{\mu}, \rho\right] e^{-\int d^{3} x \mathcal{L}}\left\langle e^{-\int d^{3} x e_{B} \rho^{2} \partial_{\mu} \gamma A^{\mu}}\right\rangle_{\gamma}
$$

and the convexity of the exponential function ensures that

$$
Z \geqslant \int D\left[A_{\mu}, \rho\right] e^{-\int d^{3} x \mathcal{L}} e^{-\int\left\langle e_{B} \rho^{2} \partial_{\mu} \gamma A^{\mu}\right\rangle} \gamma^{d^{3} x} .
$$

The average in the right-hand side vanishes (it is linear in $\gamma$ ), and the approximate partition function $Z_{p}$

$$
Z_{p}=\int D\left[A_{\mu}, \rho\right] e^{-\int d^{3} x \mathcal{L}}
$$

satisfies the variational constraint

$$
Z \geqslant Z_{p}
$$

so that the approximate free energy $\mathcal{F}_{p}=-\ln Z_{p}$ is bounded by the exact free energy $\mathcal{F}=-\ln Z$

$$
\mathcal{F}_{p} \geqslant \mathcal{F} \text {. }
$$

This bound ensures that, even neglecting the last term of the phase dependent Lagrangian $\mathcal{L}_{\gamma}$ in Eq. (16), the resulting approximate partition function $Z_{p}$ still gives a genuine variational approximation.

\section{THE GEP METHOD IN POLAR COORDINATES}

In this section we study the GEP method for the polar coordinate partition function Eq. (23) with the Lagrangian $\mathcal{L}$ defined according to Eq. (15). The GEP may be evaluated by the same $\delta$ expansion method discussed in Refs. 12 and 25 and also reported by Camarda et al. ${ }^{1}$ Here the GEP represents a variational estimate of the free energy $\mathcal{F}_{p}$.

Inserting a source term for the real field $\rho$ the partition function $Z_{p}$ reads

$$
Z_{p}[j]=\int D\left[A_{\mu}, \rho\right] e^{-\int d^{3} x \mathcal{L}} e^{-\int d^{3} x j \rho}
$$

and the free energy is given by the Legendre transform

$$
\mathcal{F}[\varphi]=-\ln Z_{p}+\int d^{3} x j \varphi
$$

where $\varphi$ is the average value of $\rho$. As usual we introduce a shifted field

$$
\widetilde{\phi}=\rho-\varphi
$$

and then we split the Lagrangian into two parts

$$
\mathcal{L}=\mathcal{L}_{0}+\mathcal{L}_{\text {int }},
$$

where $\mathcal{L}_{0}$ is the sum of two free-field terms: a vector field $A_{\mu}$ with mass $\Delta$ and a real scalar field $\widetilde{\phi}$ with mass $\Omega$

$$
\begin{aligned}
\mathcal{L}_{0}= & {\left[+\frac{1}{2}(\nabla \times \mathbf{A})^{2}+\frac{1}{2} \Delta^{2} A_{\mu} A^{\mu}+\frac{(\nabla \cdot \mathbf{A})^{2}}{2 \epsilon}\right] } \\
& +\left[\frac{1}{2}(\nabla \widetilde{\phi})^{2}+\frac{1}{2} \Omega^{2} \widetilde{\phi}^{2}\right] .
\end{aligned}
$$

The interaction then reads

$$
\begin{aligned}
\mathcal{L}_{\text {int }}= & v_{0}+v_{1} \widetilde{\phi}+v_{2} \widetilde{\phi}^{2}+v_{3} \widetilde{\phi}^{3}+v_{4} \widetilde{\phi}^{4}+\frac{1}{2}\left(e_{B}^{2} \varphi^{2}-\Delta^{2}\right) A_{\mu} A^{\mu} \\
& +e_{B}^{2} \varphi A_{\mu} A^{\mu} \widetilde{\phi}+\frac{1}{2} e_{B}^{2} A_{\mu} A^{\mu} \widetilde{\phi}^{2}
\end{aligned}
$$

where

$$
\begin{aligned}
& v_{0}=\frac{1}{2} m_{B}^{2} \varphi^{2}+\lambda_{B} \varphi^{4}, \\
& v_{1}=m_{B}^{2} \varphi+4 \lambda_{B} \varphi^{3}, \\
& v_{2}=\frac{1}{2} m_{B}^{2}+6 \lambda_{B} \varphi^{2}-\frac{1}{2} \Omega^{2}, \\
& v_{3}=4 \lambda_{B} \varphi, \\
& v_{4}=\lambda_{B} .
\end{aligned}
$$

The first-order expansion of the free energy is

$$
\begin{aligned}
\mathcal{F}[\varphi]= & \frac{1}{2} \operatorname{Tr} \ln \left[g^{-1}(x, y)\right]+\frac{1}{2} \operatorname{Tr} \ln \left[G_{\mu \nu}^{-1}(x, y)\right] \\
& +\int d^{3} x\left\{v_{0}+v_{2} g(x, x)+3 v_{4} g(x, x)^{2}\right. \\
& \left.+\frac{1}{2} e_{B}^{2}\left[g(x, x)+\varphi^{2}-\Delta^{2}\right] G_{\mu \mu}(x, x)\right\},
\end{aligned}
$$

where $g(x, y)$ is the free-particle propagator for the scalar field, and $G_{\mu \nu}(x, y)$ is the free-particle propagator for the vector field

$$
G_{\mu \nu}^{-1}(x, y)=\int \frac{d^{3} k}{(2 \pi)^{3}} e^{-i \mathbf{k}(\mathbf{x}-\mathbf{y})}\left[\delta_{\mu \nu}\left(k^{2}+\Delta^{2}\right)+\left(\frac{1}{\epsilon}-1\right) k_{\mu} k_{\nu}\right] .
$$

In the limit $\epsilon \rightarrow 0$, up to an additive constant

$$
\operatorname{Tr} \ln \left[G_{\mu \nu}^{-1}(x, y)\right]=2 \mathcal{V} \int \frac{d^{3} k}{(2 \pi)^{3}} \ln \left(k^{2}+\Delta^{2}\right),
$$

where $\mathcal{V}$ is the total volume. Dropping all constant terms, the free energy density $V_{\text {eff }}=\mathcal{F} / \mathcal{V}$ (effective potential) reads

$$
\begin{aligned}
V_{\mathrm{eff}}[\varphi]= & I_{1}(\Omega)+2 I_{1}(\Delta)+\left[\lambda_{B} \varphi^{4}\right. \\
& +\frac{1}{2} m_{B}^{2} \varphi^{2}+\frac{1}{2}\left\{m_{B}^{2}-\Omega^{2}+12 \lambda_{B} \varphi^{2}\right. \\
& \left.\left.+6 \lambda_{B} I_{0}(\Omega)\right\} I_{0}(\Omega)\right] \\
& +\left[e_{B}^{2} \varphi^{2}+e_{B}^{2} I_{0}(\Omega)-\Delta^{2}\right] I_{0}(\Delta),
\end{aligned}
$$

where the divergent integrals $I_{n}$ are defined according to

$$
I_{0}(M)=\int \frac{d^{3} k}{(2 \pi)^{3}} \frac{1}{M^{2}+k^{2}},
$$




$$
I_{1}(M)=\frac{1}{2} \int \frac{d^{3} k}{(2 \pi)^{3}} \ln \left(M^{2}+k^{2}\right),
$$

and are regularized by insertion of a finite cut-off $k<\Lambda$.

The free energy (40) now depends on the mass parameters $\Omega, \Delta$ and on the field shift $\varphi$. These are the variational parameters that must be determined by the minimization of the energy density $V_{\text {eff. }}$. At the stationary point $V_{\text {eff }}$ is the GEP and the mass parameters give the inverse correlation lengths for the fields, the so-called coherence length $\xi=1 / \Omega$ and penetration depth $\lambda=1 / \Delta$. The field shift $\varphi$ is the order parameter of the phase transition: when $\varphi \neq 0$ at the minimum of $V_{\text {eff }}$ the $U(1)$ symmetry is broken in the ground state and the system is in the superconducting phase.

The stationary conditions

$$
\begin{aligned}
& \frac{\partial V_{\text {eff }}}{\partial \Omega^{2}}=0, \\
& \frac{\partial V_{\text {eff }}}{\partial \Delta^{2}}=0,
\end{aligned}
$$

give two coupled gap equations

$$
\begin{aligned}
& \Omega^{2}=12 \lambda_{B} I_{0}(\Omega)+m_{B}^{2}+12 \lambda_{B} \varphi^{2}+2 e_{B}^{2} I_{0}(\Delta), \\
& \Delta^{2}=e_{B}^{2} \varphi^{2}+e_{B}^{2} I_{0}(\Omega) .
\end{aligned}
$$

For any $\varphi$ value the equations must be solved numerically, and the minimum point values $\Omega$ and $\Delta$ must be inserted back into Eq. (40) in order to get the Gaussian free energy $V_{\text {eff }}(\varphi)$ as a function of the order parameter $\varphi$. For a negative and small enough $m_{B}^{2}$, we find that $V_{\text {eff }}$ has a minimum at a nonzero value of $\varphi=\varphi_{\min }>0$, thus indicating that the system is in the broken-symmetry superconducting phase. Of course the masses $\Omega, \Delta$ only take their physical value at the minimum point of the free energy $\varphi_{\min }$. This point is found by requiring that

$$
\frac{\partial V_{\text {eff }}}{\partial \varphi^{2}}=0,
$$

where, as usual, the partial derivative is allowed as far as the gap Eqs. (45) and (46) are satisfied. ${ }^{11}$ The condition (47) combined with the gap Eq. (45) yields the very simple result

$$
\varphi_{\min }^{2}=\frac{\Omega^{2}}{8 \lambda_{B}} .
$$

However, we notice that here the mass $\Omega$ must be found by solution of the coupled gap equations. Thus Eqs. (48), (45), and (46) are a set of coupled equations and must be solved together in order to find the physical values for the correlation lengths and the order parameter.

Insertion of Eq. (48) into Eq. (46) yields the simple relation for the GL parameter $\kappa_{\mathrm{GL}}$

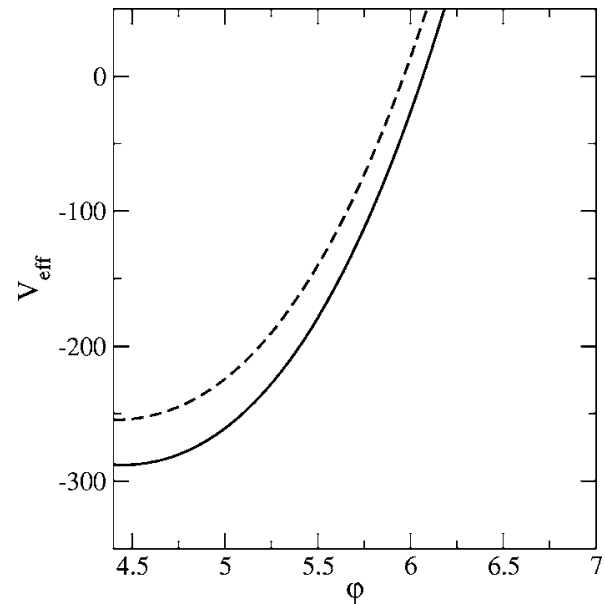

FIG. 1. Comparison between the polar GEP (solid line) and the Cartesian GEP dashed line. The value of the parameters are $m_{B}^{2} / \lambda_{B}^{2}=-80, \Lambda / \lambda_{B}=10, e_{B} / \lambda_{B}=0.001$. Note that the origin of the $\varphi$ axis coincides with the minimum of the Cartesian GEP to emphasize that our polar decomposition of the field leads to a lower effective potential.

$$
\kappa_{\mathrm{GL}}^{2}=\left(\frac{\lambda}{\xi}\right)^{2}=\kappa_{0} \frac{1}{1+\frac{I_{0}(\Omega)}{\varphi_{\min }^{2}}},
$$

where $\kappa_{0}=e_{B}^{2} /\left(8 \lambda_{B}\right)$ is the mean-field GL parameter which does not depend on temperature. As discussed by Camarda et al., ${ }^{1} \mathrm{Eq}$. (49) shows that the GL parameter is predicted to be temperature dependent through the nontrivial dependence of $\Omega$ and $\varphi_{\min }$. At low temperature, where the order parameter $\varphi_{\min }$ is large, the deviation from the mean-field value $\kappa_{0}$ is negligible. Conversely, close to the critical point, where the order parameter is vanishing, the correction factor in Eq. (49) becomes very important. The deviation from the meanfield prediction $\kappa_{\mathrm{GL}}=\kappa_{0}$ also depends on the cut-off parameter $\Lambda$ as for $\Lambda \rightarrow 0$ all the effects of fluctuations vanish together with the integrals $I_{n}$. This parameter defines another length scale $\ell=1 / \Lambda$, which is a characteristic of the material.

A test of the present method comes from the direct comparison with the Cartesian coordinate GEP discussed by Ibañez-Meier et al. ${ }^{12}$ Both methods are genuine variational methods and a direct comparison of the free-energy density gives information on the accuracy. In Fig. 1 we report our polar coordinate GEP together with the three-dimensional version of the cartesian coordinate GEP. The polar coordinate GEP yields a lower free-energy density thus indicating that, besides being more symmetric and appealing (there are no unphysical degree of freedom and no massive gauge bosons), the method is also more effective.

\section{INTERPOLATION OF THE EXPERIMENTAL DATA}

As we mentioned earlier, another appealing aspect of the GEP approximation in three space-time dimensions resides in the chance to compare its results with the phenomenology of high $T_{c}$ superconductors. For this comparison to be consistent we need to fix the bare parameters of the GL action; 


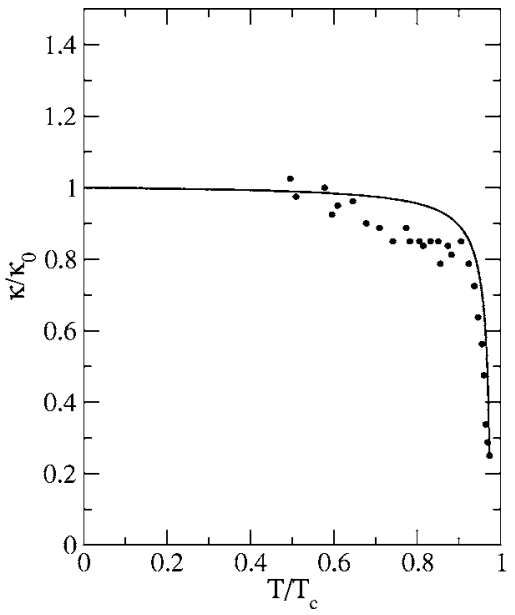

FIG. 2. The GL parameter according to Eq. (49) for $\kappa_{0}=100$, $\xi_{0}=1.36 \mathrm{~nm}, T_{c}=121.5 \mathrm{~K}$ and $\Lambda \xi_{0}=20$ (full line). The circles are the experimental data of Ref. 26 for $\mathrm{Tl}_{2} \mathrm{Ca}_{2} \mathrm{Ba}_{2} \mathrm{Cu}_{3} \mathrm{O}_{10}$.

following, ${ }^{1}$ we can use the standard derivation of the GL action (1) (see Ref. 22) to find a connection between microscopic first-principle quantities and phenomenologic bare parameters. Thus, we have

$$
e_{B}=\frac{2 e}{\hbar c} \sqrt{k_{B} T_{c} \xi_{0}},
$$

where $2 e$ represents the charge of a Cooper pair, $T_{c}$ is the critical temperature, and $\xi_{0}$ the zero temperature coherence length.

The knowledge of the zero temperature coherence length and penetration depth enables us to fix the other parameters; regarding the bare mass parameter $m_{B}^{2}$ as a linear function of temperature, we obtain

$$
m_{B}^{2}=m_{c}^{2}+\left[1-\frac{T}{T_{C}}\right]\left(m_{0}^{2}-m_{c}^{2}\right),
$$

where $m_{0}^{2}$ is the value which is required in order to find $\Omega=1 / \xi_{0}$ from the gap Eq. (45) at $\varphi=\varphi_{\min }$, and $m_{c}^{2}$ is the value of $m_{B}^{2}$ at the transition point. The mean-field approximation predicts that $m_{c}^{2}=0$, but the effect of the fluctuations is to shift the transition point to a negative nonvanishing $m_{B}^{2}$ value.

Finally, the bare coupling $\lambda_{B}$ is fixed through Eq. (46) by requiring that at zero temperature (i.e., for $m_{B}^{2}=m_{0}^{2}$ ) the penetration depth $\lambda_{0}=1 / \Delta$.

The cut-off $\Lambda$ still remains to be fixed; thus, the interpolation scheme for the superconducting properties we have to deal with contains one free parameter (i.e., $\Lambda$ ), which is a characteristic energy scale of the sample, and will be determined by a direct fit of the experimental data.

We have compared our theoretical results with the experimental GL parameter for three different materials: $\mathrm{Tl}_{2} \mathrm{Ca}_{2} \mathrm{Ba}_{2} \mathrm{Cu}_{3} \mathrm{O}_{10} \quad\left(T_{c}=121.5 \mathrm{~K}, \quad \xi_{0}=1.36 \mathrm{~nm}, \quad \kappa_{0}=100\right),{ }^{26}$ $\mathrm{YBa}_{2} \mathrm{Cu}_{2} \mathrm{O}_{7-\delta} \quad\left(T_{c}=62.2 \mathrm{~K}, \xi_{0}=1.65 \mathrm{~nm}, \kappa_{0}=187\right),{ }^{27}$ and $\mathrm{MgB}_{2}\left(T_{c}=38.6 \mathrm{~K}, \xi_{0}=3.9 \mathrm{~nm}, \kappa_{0}=31.66\right) .{ }^{28}$ For the last two materials we have obtained the GL parameter by the relation $^{29}$

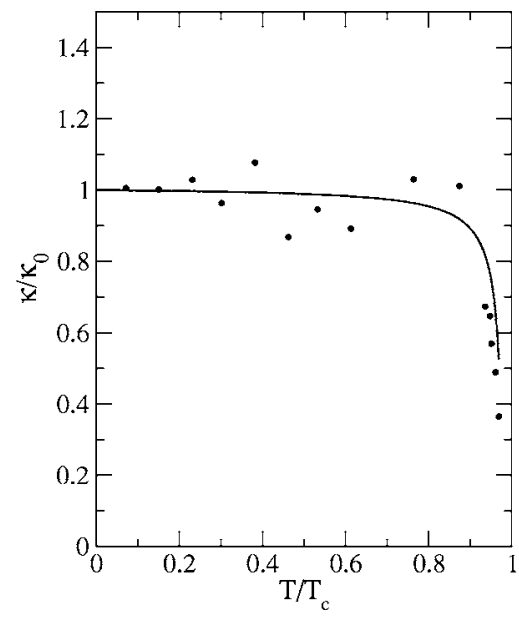

FIG. 3. The GL parameter according to Eq. (49) for $\kappa_{0}=187$, $\xi_{0}=1.65 \mathrm{~nm}, T_{c}=62 \mathrm{~K}$, and $\Lambda \xi_{0}=5$ (full line). The circles are the experimental data of Ref. 27 for $\mathrm{YBa}_{2} \mathrm{Cu}_{2} \mathrm{O}_{7} \delta$.

$$
\frac{H_{c 2}}{2 H_{c 1}}=\frac{\kappa^{2}}{\ln \kappa}
$$

(where $H_{c 1}$ and $H_{c 2}$ represent the lower and upper critical fields, respectively), and extrapolated the zero temperature phenomenological values needed to fix the bare parameters by a linear fit.

The small number of available experimental data is due to the difficulty of performing measurements of the coherence length and penetration depth (or, alternatively, of the two critical fields) up to the precritical region.

In Figs. 2-4 the comparisons between our interpolation curves, as obtained by Eq. (49), and the data relative to the three materials mentioned above, are shown. It can be noted that the experimental GL parameter is almost constant at low temperatures, according to the mean-field description, while it shows a sharp temperature dependence (at $T / T_{c} \approx 0.8$ for $\mathrm{Tl}_{2} \mathrm{Ca}_{2} \mathrm{Ba}_{2} \mathrm{Cu}_{3} \mathrm{O}_{10}$, at $T / T_{c} \approx 0.9$ for $\mathrm{YBa}_{2} \mathrm{Cu}_{2} \mathrm{O}_{7_{-} \delta}$, at $T / T_{c}$ $\approx 0.6$ for $\mathrm{MgB}_{2}$ ) in the precritical region: It is in this region

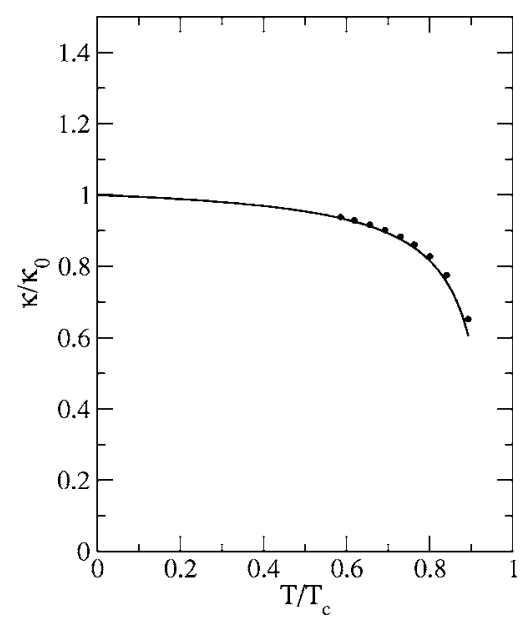

FIG. 4. The GL parameter according to Eq. (49) for $\kappa_{0}=31.66$, $\xi_{0}=3.9 \mathrm{~nm}, T_{c}=38.6 \mathrm{~K}$, and $\Lambda \xi_{0}=900$ (full line). The circles are the experimental data of Ref. 28 for $\mathrm{MgB}_{2}$. 
that the mean-field approximation breaks down, while our model shows its effectiveness. We would like to emphasize that the GEP approximation is able to reproduce the GL parameter behavior for classes of compounds (cuprates and diborides) with very different microscopic structures and, probably, different pairing mechanism. These differences are reflected in the cut-off values we used to fit the data: They have the same order of magnitude $\left(\Lambda \xi_{0}=20\right.$ for $\mathrm{Tl}_{2} \mathrm{Ca}_{2} \mathrm{Ba}_{2} \mathrm{Cu}_{3} \mathrm{O}_{10}, \Lambda \xi_{0}=5$ for $\mathrm{YBa}_{2} \mathrm{Cu}_{2} \mathrm{O}_{7} \delta$ for the two cuprates, while the cutoff used for the diboride $\left(\Lambda \xi_{0}=900\right.$ for $\mathrm{MgB}_{2}$ ) is about two order of magnitude greater. In this sense, the parameter $\Lambda$ seems to be a characteristic energy scale of the sample, related to its microscopic structure, and, as a consequence, to the size of the region where fluctuations cannot be neglected.

Finally, we want to remark that, very close to the critical point, some universal behavior should be expected and the role of thermal fluctuations becomes too important to be dealt with by the present method. In fact the GEP always predicts a weak first-order transition at the critical point even for the neutral superfluid (real scalar theory). ${ }^{30}$ This is not a problem for the interpolation of the experimatal data as the difference only arises in a very narrow range of temperature at the transition point. Thus the method seems to be suitable for interpolating the precritical region where the order of the transition does not make any difference. Any extension to the critical range would require the proper inclusion of vortex fluctuations which are expected to play a major role at criticality, ${ }^{31}$ and have been recently shown to change the order of the transition in the GL model. ${ }^{24}$ We also mention that a variational perturbative extension of the Gaussian approximation has been shown to be able to reach the critical regime. ${ }^{32}$

In summary we have shown that a polar decomposition of the complex field in the action has the merit of allowing the integration of the longitudinal vector field (by a further variational approximation), is better than the Cartesian decomposition in Ref. 12 and, at variance with the mean-field approximation, yields a one-parameter interpolation scheme which fits very well all the available data, in the whole range of the accessible temperatures. Moreover, the variational character of the model, allowing the study of any kind of coupling, makes the GEP a suitable tool for the inspection of other physical systems, such as superfluids.

As the GEP provides a nice way to interpolate the experimental data beyond the mean-field regime, we expect the method to be reliable for the description of symmetry breaking in $3+1$ dimensions where the scalar electrodynamics may be regarded as a toy model for the standard electroweak theory.

\section{ACKNOWLEDGMENT}

We are grateful to $\mathrm{H}$. Kleinert for valuable discussions and comments on the present paper.
${ }^{1}$ M. Camarda, G. G. N. Angilella, R. Pucci, and F. Siringo, Eur. Phys. J. B 33, 273 (2003).

${ }^{2}$ L. I. Schiff, Phys. Rev. 130, 458 (1963).

${ }^{3}$ G. Rosen, Phys. Rev. 172, 1632 (1968).

${ }^{4}$ T. Barnes and G. I. Ghandour, Phys. Rev. D 22, 924 (1980).

${ }^{5}$ J. Kuti (unpublished), as discussed in J. M. Cornwall, R. Jackiw and E. Tomboulis, Phys. Rev. D 10, 2428 (1974).

${ }^{6}$ S. J. Chang, Phys. Rev. D 12, 1071 (1975); Phys. Rep 23C, 301 (1975); Phys. Rev. D 13, 2778 (1976).

${ }^{7}$ M. Weinstein, S. Drell, and S. Yankielowicz, Phys. Rev. D 14, 487 (1976).

${ }^{8}$ K. Huang and D. R. Stump, Phys. Rev. D 14, 223 (1976).

${ }^{9}$ W. A. Bardeen and M. Moshe, Phys. Rev. D 28, 1372 (1983).

${ }^{10}$ M. Peskin, Ann. Phys. 113, 122 (1978).

${ }^{11}$ P. M. Stevenson, Phys. Rev. D 32, 1389 (1985).

${ }^{12}$ R. Ibañez-Meier, I. Stancu, and P. M. Stevenson, Z. Phys. C 70, 307 (1996).

${ }^{13}$ D. S. Fisher, M. P. A. Fisher, and D. A. Huse, Phys. Rev. B 43, 130 (1991).

${ }^{14}$ T. Schneider and D. Ariosa, Z. Phys. B: Condens. Matter 89, 267 (1992).

${ }^{15}$ T. Schneider and H. Keller, Int. J. Mod. Phys. B 8, 487 (1993).

${ }^{16}$ S. Kamal, D. A. Bonn, N. Goldenfeld, P. J. Hirschfeld, R. Liang, and W. N. Hardy, Phys. Rev. Lett. 73, 1845 (1994).

${ }^{17}$ T. Schneider, R. Khasanov, K. Conder, E. Pomjakushina, R. Bruetsch, and H. Keller, J. Phys.: Condens. Matter 16, L1 (2004).
${ }^{18}$ T. Schneider, R. Khasanov, and H. Keller, Phys. Rev. Lett. 94, 077002 (2005).

${ }^{19}$ I. Herbut and Z. Tesanovic, Phys. Rev. Lett. 76, 4588 (1996).

${ }^{20}$ L. M. Abreu, A. P. C. Malbouisson, and I. Roditi, Eur. Phys. J. B 37, 515 (2004).

${ }^{21}$ See Ref.22, Vol. I, Part II, Sec. 3.11.

${ }^{22}$ See for instance, H. Kleinert, Gauge Fields in Condensed Matter (World Scientific, Singapore, 1989).

${ }^{23}$ B. S. De Witt, Phys. Rev. 162, 1195, 1239 (1967); L. D. Faddeev, V. N. Popov, Phys. Lett. 25B, 29 (1967).

${ }^{24}$ H. Kleinert, cond-mat/0509430 (unpublished).

${ }^{25}$ I. Stancu and P. M. Stevenson, Phys. Rev. D 42, 2710 (1990).

${ }^{26}$ G. Brandstatter, F. M. Sauerzopf, H. W. Weber, F. Ladenberger, and E. Schwarzmann, Physica C 235-240, 1845 (1994).

${ }^{27}$ K. G. Vandervoort, U. Welp, J. E. Kessler, H. Claus, G. W. Crabtree, W. K. Kwok, A. Umnezawa, B. W. Veal, J. W. Downey, A. P. Paulikas, and J. Z. Liu, Phys. Rev. B 43, 13042 (1991).

${ }^{28}$ M. Xu, H. Kitazawa, Y. Takano, J. Ye, K. Mishida, H. Abe, A. Matsushita, N. Tsujii, and G. Kido, Appl. Phys. Lett. 79, 2779 (2001).

${ }^{29}$ C. P. Poole, H. A. Farach, and R. J. Creswick, Superconductivity (Academic Press, New York, 1995).

${ }^{30}$ F. Siringo and L. Marotta, Eur. Phys. J. C 44, 293 (2005).

${ }^{31}$ M. Camarda, F. Siringo, R. Pucci, and A. Sudbo, (unpublished).

${ }^{32}$ H. Kleinert and V. Schulte-Frohlinde, Critical Properties of $\Phi^{4}$ Theories (World Scientific, Singapore, 2001). 